it is a text-book, and its role is to give the student the thermodynamic background required by a degree course and to be an introduction to more advanced treatises. Part I covers the first two laws of thermodynamies, dilute solutions and electrochemistry; Part 2, with entropy, free energy, the third law, solutions and thermodynamic functions, is designed to be studied as a second course.

There are two new chapters. One is an introduction to the thermodynamics of irreversible processes (such as transport across membranes), and the other (by Dr. D. J. R. Laurence) is on the thermodynamics of polymers, and discusses the extensions necessary to account for the properties of polymer solutions, in which entropy considerations are naturally dominant, and the consequences which occur when solvent and solute are of a different order of molecular magnitude.

D. M. Russeli

\section{Pyridine and its Derivatives}

Part 3. Edited by Erwin Klingsberg. (The Chemistry of Heterocyclic Compounds: a Series of Monographs, Vol. 14, Part 3.) Pp. ix +914 . (New York and London: Interscience Publishers, a Division of John Wiley and Sons, 1962.) 488s.

THIS, the third and largest, part follows the format of the previous two and comprises four chapters on some functional derivatives of pyridine.

A. S. Tomeufcik and L. N. Starker contribute to the first chapter (177 pp.) on aminopyridines. References to infra-red studies relating to the structure of these compounds are quoted up to 1952 . Perhaps some note might have been included here to the later contributions made by Katritzky et al.

The next two chapters are devoted to carboxylic acids. The first is by E. P. Oliveto and includes an excellent account of their preparation reactions and functional derivatives. The chapter concludes with a short account of some aspects of the naturally occurring derivatives of this group in vitamins and alkaloids. The second chapter on acids, by J. C. Godfrey, is concerned with the carboxylate group as a side-chain in the pyridine nucleus. The subject material is given the same systematic treatment as before.

Pyridinols and pyridones, which are important intermediates in the armamentarium of the synthetic organic chemist, are adequately dealt with in the last chapter (382 pp.) by H. Meislich. Tho aforementioned chapters aro remarkably free from errors and each is well supplemented with tables and a comprehensive bibliography. This is a most useful contribution to the chemistry of pyridine and maintains the high standard of the series.

The high cost precludes its purchase by the vocational organic chemist, and any browsing involves the borrowing for the night or week-end from the works or academic reference library.

R. HULL

\section{Spectrometric Identification of Organic Compounds}

By Dr. Robert M. Silverstein and Dr. G. Clayton Bassler. Pp. viii +177 . (Now York and London: John Wiley and Sons, Inc., 1963.) 64s.

THIS is in many ways an unusual, but welcome, scientific publication. The strongly didactic character of its presentation will appeal particularly to advanced students who are anxious to improve their knowledge of modern instrumental techniques and of their diverse applications.

The book is of quarto size, the text printed legibly on good paper, and the tables and diagrammatic illustrations are all that one could reasonably expect. Four earlier chapters are individually devoted to the fairly detailed study of each of four spectrometric methods. The older, rugged techniques of infra-red and ultra-violet spectrometry are considered side-by-side with the more complex modern instrumental systems concerned with nuclear magnetic resonance and fragment mass spectroscopy. The finer details of instrument design are left to more authoritative text-books, but sufficient information is given to provide the tyro with a clear picture of the functioning of a particular instrument and of the theoretical implications of the recorded, data which it provides.

In later chapters, the authors illustrate the powerful part which these techniques can play in the unequivocal identification of small (but pure) amounts of various organic compounds. Tetramerous analytical exercises have been carried out with these instruments on various organic substances. All the experimental data are clearly presented, the physical clues are marshalled and critically examined. Conclusions are then drawn with a Holmosian precision and provide a composite molecular and structural diagnosis which could well excite the admiration of sterner and less-gullible observers than the susceptible Dr. Watson. Quantities of less than one milligram of unknown substance are adequate for all techniques with the exception of nuclear magnetic resonance; and only in the special instance of fragment mass spectroscopy does the original sample under examination become irrecoverable.

Tables of numerical data are given which greatly simplify the interpretation of the many quatern spectra which the authors have provided for twenty organic compounds of various types. Thus 26 pages are devoted to J. H. Benyon's tables for the masses and isotopic abundance ratios for various combinations of carbon, hydrogen, oxygen and nitrogen; that is, values of the 100 per cent parent peak " $P$ " and of $(P+1)$ and $(P+2)$. Similarly useful tabular data are provided for the other spectrometric disciplines described in this work. The final chapter provides 10 sets of quatern spectra with Beilstein references, a most useful complementary student exercise.

This book is reasonably priced and is certain to attract the attention of those who wish to revise their knowledge of the older techniques and to keep abreast of the surging tide of modern physical science.

D. T. LEWIS

\section{Les Cyclitols}

Chimie, Biochimie, Biologie. Par Prof. Théodore Posternak. (Chimie des Substances Naturelles.) Pp. 492. (Paris: Hermann, 1962.) 48 N.F.

THIS volume, one of the admirable series on the chemistry of natural products, deals with the monocyclic polyhydric alcohols in general, but its main concern is with the inositols and derived substances. These are, of course, the most important of the natural cyclitols and constitute a large group of compounds which, however, are chemically relatively simple. These range from the unsubstituted hexahydroxycyclohexanes through methylated derivatives to the complex lipids, the phosphoinositides. Meso(myo)-inositol is a growth factor for many organisms including mammals, although its status as a vitamin, in man, is disputed.

Prof. Posternak has devoted much of his research career to the chemistry and biochemistry of these com. pounds, and has now, fittingly, produced this scholarly work in which every aspect of the subject is discussed in a detailed but readable fashion. The book is timely, because much of the chemistry is now well understood as a result of work in the intervening years since the previous major work in this field was published some fifteen years ago. On the contrary, however, the biological involvement of these substances is not at all clear and one may expect that the book will give added stimulus to further work.

This soft-covered, but sturdy, volume is beautifully produced. References include those to work published in 1960, but a number of pages of addenda at the end of the book discuss more recent work. It should find its way on to the shelves of all chemical and biochemical libraries.
D. M. BRown 